\title{
Rediminds Entry in KiTS19 Competition
}

\author{
Hamid Ali $^{\mathrm{a}}$, Trevor Zeffiro ${ }^{\mathrm{a}}$, Anubhav Reddy Nallabasannagari ${ }^{\mathrm{a}}$, Madhu \\ Reddiboina $^{\mathrm{a}}$, Mahendera Bhandari ${ }^{\mathrm{a}, \mathrm{b}}$ \\ ${ }^{a}$ Rediminds Research, Southfield Michigan \\ ${ }^{b}$ Vatticuti Foundation
}

\begin{abstract}
Since its presentation in 2015, U-Net has been rapidly adopted by the medical imaging community. However, due to the large number of interrelated parameters, different implementations of preprocessing, architechture, and training can result in wildly different levels of performance, thus, establishing a baseline by which we can compare the results of different methods. As such, in our submission we submit the results of high-resolution 3 dimensional segmentation of kidney and tumor using NN-Unet.
\end{abstract}

Keywords: Semantic Segmentation, U-Net, Urology, Medical Imaging

\section{Introduction}

Due to the prevalence of Renal Cell Carcinoma, among other forms of kidney cancer, and advances in other branches of medicine, such as Ultrasound-guided liver surgery[1], a great amount of effort has been allocated to the better visualization, and segmentation of kidney and tumor from the surrounding tissue. With sufficiently accurate tumor segmentation, and existing methods of tracking surgical implements, the opportunity to reduce patient complications though less invasive procedures should not be overlooked.

The KiTS19 Grand Challenge Competition, is such an endeavor; through the accurate segmentation kidney from cancer, in an automated fashion, less invasive methods could be applied more easily to patients. Data from the KiTS19 Grand Challenge was provided in the NIFTI format, with characterization of the spatial resolution of the CT scan, also provided therein. Of the 300 cases provided, 210 were contained both imaging, and segmentation data, and 90 contained only imaging data. These 300 cases were selected from patients who had undergone either a partial, or radical nephrectomy at the University of Minnesota Medical Center, and who had a preoperative arterial phase $\mathrm{CT}$. 


\section{Methods}

\subsection{Data Preparation and Augmentation}

Insofar as data augmentation, we used the default data-preprocessing from NNU-Net[2], as well as the models included therein. As such, CT scans were cropped to only relevant regions for kidney segmentation, normalized with respect to voxel size, and intensity, and randomly rotated by angles $\alpha, \beta$ about the $\mathrm{x}$ and $\mathrm{z}$ axis such that $|\alpha|,|\beta| \leq \frac{\pi}{12}$. Furthermore, random tweaks were made to voxel dimensions, and the $\gamma$ of each scan

\subsection{Training and Optimization}

We trained the model with cross-entropy loss, and the ADAM optimizer with an initial learning rate of $3 * 10^{-4}$. If the loss did not decrease after 25 epochs, training was ended. Training was done using 2 of the 5 folds in 5 -fold cross validation, completed by randomly assigning each case to into one of five groups. Each of these groups can then be utilized as a test set, and then the remaining 4 can be used for training. In our case however, our results did not change much after the second fold, thus, we ended training there.

\subsection{Data Post processing}

The inferences were generated by considering several three dimensional patches of size $[192,192,48]$, and then stitching these inferences back together.

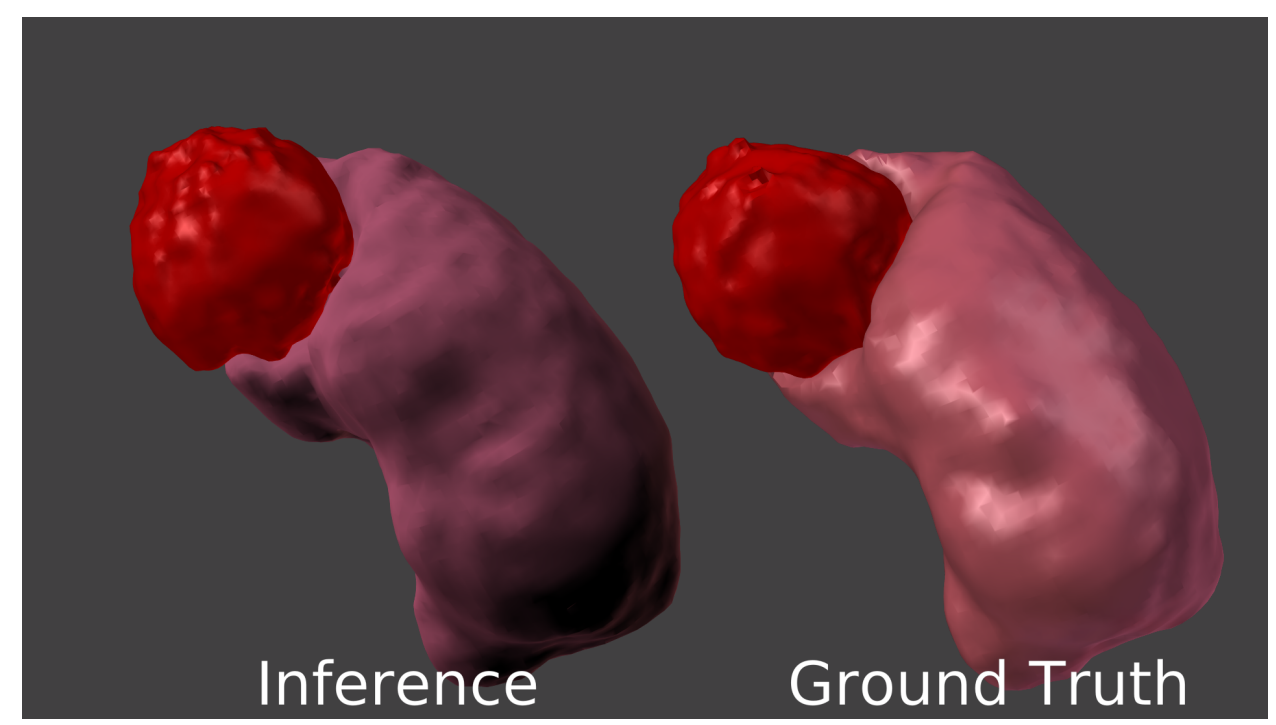

Figure 1: A rendering of the output of our model, compared to a rendering of the ground truth 


\section{Results}

Below are the resulting performance metrics on the provided KiTS19 test Dataset. A comparison between a 3 dimensional rendering of our model's output and the ground truth is depicted in Figure 1.

Table 1: Results of model on the Test Dataset

\begin{tabular}{lc} 
Object & Dice Coefficient \\
\hline Kidney & .9390 \\
Tumor & .7094 \\
\hline Combined & .8242 \\
\hline
\end{tabular}

\section{Limitations}

We likely could achieve better results by completing all 5 folds of 5 -fold cross-validation, training additional models, such as a 3D cascade, and $2 \mathrm{~d}$ segmentation on CT slices, and then ensembling the results, however due to long training times, we did not pursue such an approach.

\section{References}

[1] A. L. Simpson, T. P. Kingham, Current evidence in image-guided liver surgery, Journal of Gastrointestinal Surgery 20 (6) (2016) 1265-1269. doi:10.1007/s11605-016-3101-7.

URL https://doi.org/10.1007/s11605-016-3101-7

[2] F. Isensee, J. Petersen, A. Klein, D. Zimmerer, P. F. Jaeger, S. Kohl, J. Wasserthal, G. Koehler, T. Norajitra, S. Wirkert, K. H. Maier-Hein, nnu-net: Self-adapting framework for u-net-based medical image segmentation (2018). arXiv:1809.10486. 